\title{
The role of intramineral proteins involved into the biomineralization of calcium carbonate in eggshells formation. Implications to the dinosaurs' extinction
}

\author{
Abel Moreno and Nerith Rocío Elejalde-Cadena \\ Instituto de Química, Universidad Nacional Autónoma de México. Avenida Universidad 3000. Colonia UNAM. Alcaldía Coyoacán. \\ 04510 Ciudad de México, México. \\ carcamo@unam.mx
}

In this talk, the role that intramineral proteins have played on the shape control as well as in the biomineralization of calcium carbonate in the eggshell's formation of different avian, crocodiles and dinosaurs will be reviewed. Particularly, the collected eggshells samples of five fossilized eggshells from dinosaurs that roamed the Earth more than 65 million years ago. We characterized the eggshells of the Theropod (bipedal carnivores) and Hadrosauridae (duck-billed dinosaurs) families and an unidentified ootaxon. We have found the existence of some proteins by using micro X-ray absorption and micro-fluorescence techniques at the synchrotron facilities. From these analyses on the dinosaur eggshells, X-ray absorption methods showed a very characteristic organic sulfur bonding similar to that semi-essential proteogenic amino acid L-cysteine, which implies that there is a possibility of having a very old intramineral protein similar to those found in emu and crocodiles. On the other hand, the spectroscopical characterization on these samples showed that calcium carbonate was the primary mineral, with smaller amounts of albite and quartz crystals. Anhydrite, hydroxyapatite, and iron oxide impurities were also present in the shells, which suggests replacement of some of the original minerals during fossilization. Then, with Fourier transform infrared spectroscopy (FT-IR), we found nine amino acids among the five samples, being lysine the only amino acid present in all of them. In addition, we have found evidence of secondary protein structures, including turns, $\alpha$-helices, $\beta$-sheets and disordered structures, which have been preserved for millions of years by being engrained in the minerals. The FT-IR bands corresponding to amino acids and secondary structures could be indicative of ancestral proteins that have not been characterized before. This type of chemical, spectroscopical and structural characterization together with the optical one is a relevant contribution to the field of biomineralization of calcium carbonate research, mainly because these types of samples are unique in their type due to the biological relevance in Mexico and will, therefore, allow us to understand the species that became extinct millions of years ago as well as the importance of calcium carbonate associated to ancient proteins throughout the biomineralization processes on Earth.

Keywords: calcium carbonate; biomineralization, intramineral proteins, eggshells, characterization 